\section{OPEN ACCESS}

Edited by:

Sebastian Cerdan

Consejo Superior de Investigaciones

Científicas (CSIC), Spain

Reviewed by:

Yanwei Miao,

Dalian Medical University, China

Jesus Pacheco Torres,

Johns Hopkins University,

United States

*Correspondence:

Eleni Gkika

eleni.gkika@uniklinik-freiburg.de

Specialty section:

This article was submitted to

Cancer Imaging and Image-directed

Interventions,

a section of the journal

Frontiers in Oncology

Received: 02 December 2019

Accepted: 08 June 2020

Published: 12 August 2020

Citation:

Gkika E, Benndorf M, Oerther B, Mohammad F, Beitinger S, Adebahr S,

Carles M, Schimek-Jasch T,

Zamboglou C, Frye BC, Bamberg $F$

Waller CF, Werner M, Grosu AL,

Nestle U and Kayser G (2020) Immunohistochemistry and Radiomic

Features for Survival Prediction in Small Cell Lung Cancer.

Front. Oncol. 10:1161.

doi: 10.3389/fonc.2020.01161

\title{
Immunohistochemistry and Radiomic Features for Survival Prediction in Small Cell Lung Cancer
}

\begin{abstract}
Eleni Gkika ${ }^{1 *}$, Matthias Benndorf ${ }^{2}$, Benedict Oerther ${ }^{2}$, Farid Mohammad ${ }^{2}$, Susanne Beitinger ${ }^{3}$, Sonja Adebahr ${ }^{1,4,5}$, Montserrat Carles ${ }^{1}$, Tanja Schimek-Jasch ${ }^{1}$, Constantinos Zamboglou ${ }^{1}$, Björn C. Frye ${ }^{6}$, Fabian Bamberg ${ }^{2,7}$, Cornelius F. Waller ${ }^{7,8}$, Martin Werner ${ }^{7,9}$, Anca L. Grosu ${ }^{4,5,7}$, Ursula Nestle ${ }^{4,5,7,10}$ and Gian Kayser ${ }^{9}$

${ }^{1}$ Department of Radiation Oncology, Medical Center, University Hospital Freiburg, Freiburg, Germany, ${ }^{2}$ Department of Radiology Freiburg, University Medical Center Freiburg, Freiburg, Germany, ${ }^{3}$ Department of Neurology, Medical Center, University Hospital Freiburg, Freiburg, Germany, ${ }^{4}$ German Cancer Consortium (DKTK), Freiburg, Germany, ${ }^{5}$ German Cancer Research Center (DKFZ), Heidelberg, Germany, ${ }^{6}$ Department of Pneumology, Medical Center, University Hospital Freiburg, Freiburg, Germany, ${ }^{7}$ Faculty of Medicine, University of Freiburg, Freiburg, Germany, ${ }^{8}$ Department of Hematology, Oncology and Stem Cell Transplantation, University Medical Center Freiburg, Freiburg, Germany, ${ }^{9}$ Department of Pathology, Faculty of Medicine, Medical Center, Institute of Surgical Pathology, University Hospital Freiburg, Freiburg, Germany, ${ }^{10}$ Department of Radiation Oncology, Kliniken Maria Hilf, Mönchengladbach, Germany
\end{abstract}

Background: The aim of the study was to evaluate the role of different immunohistochemical and radiomics features in patients with small cell lung cancer (SCLC).

Methods: Consecutive patients with histologically proven SCLC with limited ( $n=47$, $48 \%$ ) or extensive disease ( $n=51,52 \%)$ treated with radiotherapy and chemotherapy at our department were included in the analysis. The expression of different immunohistochemical markers from the initial tissue biopsy, such as CD56, CD44, chromogranin A, synaptophysin, TTF-1, GLUT-1, Hif-1a, PD-1, and PD-L1, and MIB-1/KI-67 as well as LDH und NSE from the initial blood sample were evaluated. $\mathrm{H}$-scores were additionally generated for CD44, Hif-1a, and GLUT-1. A total of 72 computer tomography (CT) radiomics texture features from a homogenous subgroup $(n=31)$ of patients were correlated with the immunohistochemistry, the survival (OS), and the progression-free survival (PFS).

Results: The median OS, calculated from diagnosis, was 21 months for patients with limited disease and 13 months for patients with extensive disease. The expression of synaptophysin correlated with a better OS (HR 0.546 95\% Cl 0.308-0.966, $p=0.03$ ). The expression of TTF-1 (HR 0.286, 95\% Cl: 0.117-0.698, $p=0.006)$ and a lower GLUT-1 H-score (median = 50, HR: 0.511, 95\% Cl: 0.260-1.003, $p=0.05$ ) correlated with a better PFS. Patients without chromogranin A expression had a higher risk for developing cerebral metastases $(p=0.02)$ and patients with PD 1 expression were at risk for developing metastases $(p=0.02)$. Our radiomics analysis did not reveal a single texture feature that correlated highly with OS or PFS. Correlation coefficients ranged between -0.48 and 0.39 for OS and between -0.46 and 0.38 for PFS. 


\begin{abstract}
Conclusions: The role of synaptophysin should be further evaluated as synaptophysin-negative patients might profit from treatment intensification. We report an, at most, moderate correlation of radiomics features with overall and progression free survival and no correlation with the expression of different immunohistochemical markers.
\end{abstract}

Keywords: SCLC, immunohistochemistry, radiotherapy, radiomics, biomarkers, synaptophysin

\section{INTRODUCTION}

SCLC is an aggressive, high-grade neuroendocrine tumor associated with a short doubling time, a high growth fraction, and early development of widespread metastases, which contribute to the extremely poor prognosis of the disease (1-3). In an era of precision medicine, there is an increasing need for defining biomarkers that could assist in therapeutic decision making. Immunohistochemistry (IHC) is a widely available technique that can provide quickly and cost-efficiently clinically significant results in terms of diagnosis. Although some of the cytomorphologic features of small cell lung cancer (SCLC) are well-defined in the literature, little is known about their predictive or prognostic value.

Currently, synaptophysin, chromogranin A, and CD 56 are neuroendocrine markers routinely used for the diagnosis of SCLC $(4,5)$. However, $10-15 \%$ of the neuroendocrine markers have variable expressions or lack expression (6-8). Pulmonary SCLC show thyroid transcription factor-1 (TTF-1) expression in more than $90 \%(9,10)$ similar to CD56, which stains $\sim 90-$ $100 \%$ of the cases. In addition, $25-37 \%$ of SCLC cells show a positive staining for CD $44(11,12)$ and about $78-92 \%$ of the patients show a glucose transporter 1 (GLUT-1) expression (1315). Tumors with a rapid doubling time typically have a high mitotic rate and a proliferation index of $70-90 \%(5,8,16)$. The role of hypoxia-inducible factor 1a (HIF-1a) in SCLC is not well-defined. Wan et al. suggest that HIF-1a may enhance the angiogenic potential of SCLC by regulating some angiogenic genes, such as the vascular endothelial growth factor VEGF-A (17), but literature is limited. Programmed cell death ligand 1 (PD-L1) is a predictive biomarker for immunotherapy in several solid tumors, but its role in the treatment of SCLC is not welldefined (18-22).

It has been suggested that the intra-tumoral heterogeneity within solid cancers could be captured with the use of medical imaging, which might enhance our understanding of the tumor biology $(23,24)$. The use of radiomics might provide additional information to the immunohistochemistry concerning the phenotype of the tumor. In this analysis, we evaluate the potential role of different CT radiomic features and immunohistochemical markers, such as CD56, chromogranin A, synaptophysin, TTF1, Ki 67, CD44, HIF-1a, GLUT-1, PD 1, and PD-L1, in patients with SCLC.

\section{METHODS AND MATERIALS}

\section{Patient Characteristics}

The study was approved by the local ethics committee. Consecutive routine patients with histologically proven SCLC receiving radiation therapy and chemotherapy at our department were included in this study. Computer tomographies (CTs) of the thorax and abdomen, bone scans, as well as magnetic resonance imaging (MRI) of the brain were performed during the initial routine workup. Tumors were staged and assessed according to the Union Internationale Contre le Cancer (UICC) seventh edition.

Patients were routinely evaluated during treatment. Complete blood tests, including blood count, and biochemical analysis, such as liver and renal function tests, lactate dehydrogenase (LDH, [U/l], Supplementary Table 1) and tumor markers, such as neuron-specific enolase (NSE, $[\mu \mathrm{g} / \mathrm{l}]$, Supplementary Table 1) were assessed routinely. Follow-up visits were scheduled every 3 months, including physical examination and CTs. Tumor response was assessed according to the Response Evaluation Criteria In Solid Tumors (RECIST) criteria (25).

\section{Treatment Characteristics}

Chemotherapy typically consists of four to six cycles of cisplatinbased doublets (or carboplatin in the presence of extensive disease or comorbidities) in combination with etoposide administered every 21 days. In the absence of metastases, chemoradiation (50.4 to $66 \mathrm{~Gy}$ in $1.8-2$ Gy daily fractions) was delivered concurrently with the second cycle of chemotherapy. In case of symptom palliation, 30-36 Gy in 3-Gy daily fractions were delivered. Patients without cerebral metastases with or without distant metastases who responded after initial treatment received prophylactic cranial irradiation (PCI) up to a total dose of $30 \mathrm{~Gy}$, according to national guidelines.

\section{Immunohistochemistry}

Formalin-fixed paraffin-embedded tissue samples were cut into $3-\mu \mathrm{m}$ slices and mounted on positively charged glass slides. Immunohistochemistry was performed using a Dako Autostainer Plus-Link (Dako, Glostrup, Denmark). Details of the antibodies and staining protocols used are listed in Supplementary Table 2.

For all antibodies, specific staining patterns [i.e., nuclear for KI67, TTF-1, membranous for CD56, GLUT1, PD-L1 (tumor cells, macrophages, stroma) and cytoplasmic for CD 44, Chromogranin, PD-1] were evaluated. Any intensity was recorded. TTF-1, CD56, Chromogranin A, and Synaptophysin were classified as positive if the majority of tumor cells showed specific staining reactivity.

For CD44, GLUT-1, and HIF-1a, H-scores were calculated according to the following formula $(26,27)$ :

$[1 \times(\%$ cells $1+)+2 \times(\%$ cells $2+)+3 \times(\%$ cells $3+)]$.

All viable tumor cells within the biopsy were taken into account when applying the semi-quantitative scoring system. 


\section{Radiomics Subgroup Analysis}

We performed a radiomics analysis in a homogenous patient subgroup, concerning the CT scans, performed either with a Siemens Sensation 16 or a Siemens Sensation 64 scanner $(n=31$, $n=13$ at Siemens Sensation 16, $n=18$ at Siemens Sensation 64). All patients received intravenous contrast agent (mean: $82 \mathrm{ml}$, range: $60-100 \mathrm{ml}$, similar in both scanners; $p=0.93$, two-sided $t$ test). Images were analyzed in 3-mm lung kernel reconstructed slices. All images had a matrix of $512 \times 512$ voxels. Comparison (two sided $t$ test) of mean HU (and standard deviation, SD) of segmented tumors between the two scanners to identify a possible systematic bias of gray values analyzed resulted in a $p$-value of 0.45 (0.07 SD).

The primary tumor was predefined by a radiation oncologist, and three-dimensional, semi-automated segmentation was performed by a radiologist in the axial images. Segmentation was performed in a 3-D slicer (28), using the grow-cut algorithm (29). This algorithm has been demonstrated to generate more robust segmentation results than manual definition of tumor volume for NSCLC cases (29). A radiologist manually sets seeds within the tumor and in the surrounding different tissues, e.g., mediastinum, lung, and chest wall. The algorithm then grows regions of interest that fit best to the set seeds according to voxel gray value (Hounsfield units). Results were inspected and, in case of obvious flaws, were manually corrected by the radiologist. An example of the semiautomated tumor segmentation is provided in Figure 1.

We employed the radiomics pipeline implemented in the PyRadiomics package for Python to derive texture features (30). A total of 72 texture features was obtained for each tumor (all features from the implemented classes gray level co-occurrence matrix, gray level run length matrix, first order histogram features, and shape features were derived). We calculated correlation coefficients (Pearson correlation and Spearman correlation to account for possible non-linear associations) for the single texture features with overall survival and progressionfree survival.

\section{Statistical Analysis}

Overall survival (OS) was calculated as time from pathological confirmation until death from any cause with censoring at the date last seen alive. Progression-free survival (PFS) was calculated as time from start of treatment until death or documentation of progression. OS and PFS were estimated by the Kaplan-Meier method. The Cox proportional hazards regression model was used for further analyses of possible prognostic factors for OS and PFS. All immunohistochemical markers were regarded as categorical variables, $\mathrm{H}$ scores, MIB-1, NSE, and LDH were regarded as continuous variables and were reported as median with the corresponding range (minimum and maximum). Univariate and multivariate analysis was performed with the Cox proportional hazards model. For multivariate analysis, we included in the model only the parameters that were statistically significant in the univariate analysis. Results are reported as hazard ratios (HR), 95\% confidence intervals (CI), and $p$-values.

Concerning radiomics analysis, the Pearson and Spearman correlation coefficients were used for correlation of single texture features with OS and PFS to account for possible non-linear associations. Due to the small sample size and general recommendation to incorporate around 20 cases per explanatory variable in multivariate regression models (31), we build bivariate Cox proportional-hazard models with the two radiomics features correlated most with OS

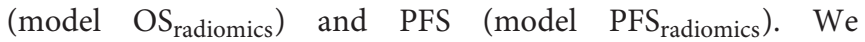
required features to be moderately autocorrelated at most. Furthermore, we build bivariate models with the radiomics feature that exhibits strongest correlation with OS/PFS and NSE and LDH serum level as additional variables (models OS radiomics-NSE, OS radiomics-LDH, PFS $_{\text {radiomics-NSE, and }}$ $\mathrm{PFS}_{\text {radiomics-LDH. Univariate models are generated for all }}$ models for comparison.

The statistical significance level was set at $\leq 0.05$. All $p$-values were two-sided. Statistical analysis was performed using SPSS version 23 (SPSS, Chicago IL).

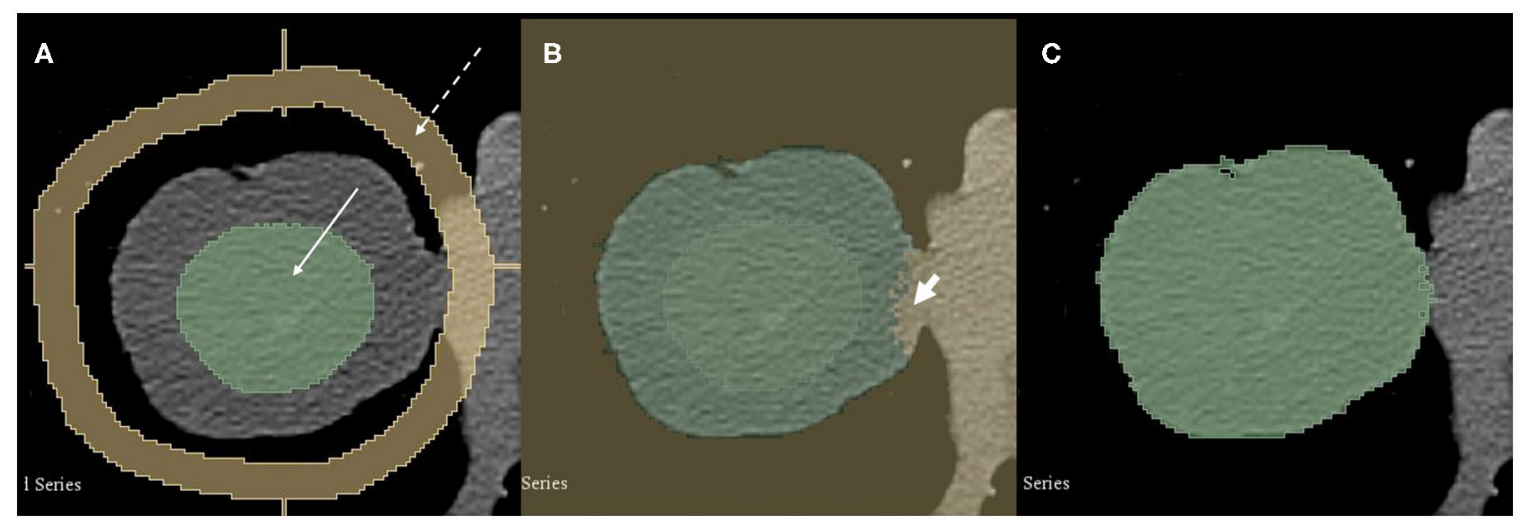

FIGURE 1 | A 74-year-old male patient with pT2 pNO cMO RO G3 SCLC in the middle lobe. Semi-automated segmentation process. (A) A radiologist manually set seeds in the tumor (green, solid arrow) and the surrounding tissue (yellow, dashed arrow). (B) The grow-cut algorithm is applied and automatically segments the tumor. Note small error on the medial side (short arrow). Such errors are corrected manually. (C) Final tumor segmentation. 


\section{RESULTS}

\section{Patient and Treatment Characteristics}

Consecutive patients $(n=101)$ with evaluable pathological specimens $(n=98)$ in our pathology department treated in the radiation oncology department between 2004 and 2014 were included in the analysis. A disseminated disease was present at diagnosis in $51(52 \%)$ patients, and $47(48 \%)$ of the patients had no metastatic disease. Patient characteristics are shown in Table 1.

A total of 70 patients received a thoracic radiotherapy, of which 55 (56\%) patients (M0 or M1a) were treated in curative intent with a median dose of $50.4 \mathrm{~Gy}$ and 15 patients for palliation with a median dose of $30 \mathrm{~Gy}$. A total of 50 patients with good response after initial treatment, of which 37 had no signs of metastases and 13 with extracranial metastatic spread at diagnosis, received a prophylactic cranial irradiation and 31 patients a palliative whole brain irradiation due to cerebral metastases. Fifty-five

\section{TABLE 1 | Patient and treatment characteristics.}

\begin{tabular}{|c|c|}
\hline Variable & $\mathrm{Nr}(\%)$ or median (range) \\
\hline Age (years) & $64(42-84)$ \\
\hline \multicolumn{2}{|l|}{ Gender } \\
\hline Male & $63(64 \%)$ \\
\hline Female & 35 (36\%) \\
\hline \multicolumn{2}{|l|}{ TNM } \\
\hline \multicolumn{2}{|l|}{$\mathrm{T}$} \\
\hline $\mathrm{T} 1$ & 7 (7\%) \\
\hline $\mathrm{T} 2$ & $15(15 \%)$ \\
\hline T3 & $23(24 \%)$ \\
\hline $\mathrm{T} 4$ & 53 (54\%) \\
\hline \multicolumn{2}{|l|}{$N$} \\
\hline NO & $10(10 \%)$ \\
\hline N1 & $6(6 \%)$ \\
\hline N2 & $31(32 \%)$ \\
\hline N3 & $51(52 \%)$ \\
\hline \multicolumn{2}{|l|}{ M } \\
\hline MO & 47 (48\%) \\
\hline M1 & $51(52 \%)$ \\
\hline \multicolumn{2}{|l|}{ Stadium } \\
\hline I & $4(4 \%)$ \\
\hline$\|$ & $6(6 \%)$ \\
\hline III & 37 (38\%) \\
\hline IV & 51 (52 \%) \\
\hline \multicolumn{2}{|l|}{ ECOG } \\
\hline 1 & $60(61 \%)$ \\
\hline 2 & 35 (36\%) \\
\hline n.s & 3 (3\%) \\
\hline NSE [ $\mu \mathrm{g} / 1]$ & $42.2(10.8-597.0)$ \\
\hline $\mathrm{LDH}[\mathrm{U} / \mathrm{I}]$ & $258(146-973)$ \\
\hline
\end{tabular}

ECOG, Eastern Cooperative Oncology Group; NSE, neuron-specific enolase; $L D H$, lactate dehydrogenase.
(56\%) patients received Carboplatin/Etoposide, and 30 (31\%) patients Cisplatin/Etoposide. One patient did not receive any chemotherapy due to comorbidities, and 11 patients received different platinum-doublets in combination with topotecan $(2 \%)$ or paclitaxel and etoposide $(2 \%)$ or pemetrexed $(3 \%)$ or analog to the EPICO protocol (epirubicin/cyclophosphamid/vincristin, $4 \%)$. One patient received topotecan alone.

\section{Overall Survival and Progression-Free Survival}

The median follow-up was 73 months for patients alive. The median overall survival for the whole group was 15 months (95\% CI: 12-18). The median survival of the patients without metastases was 21 (95\% CI: 16-28) months and for patients with distant metastases 13 (95\% CI: 11-15) months, respectively. The OS at 2 and 5 years was 33 and 13\% for the patients without metastases and 14 and $0 \%$ for patients with metastatic disease, respectively. The median progression-free survival was 7 months.

Patients with higher LDH (HR 1.003, 95\% CI 1.001-1.004, $p$ $<0.0001$ ) and NSE (HR 1.004, 95\% CI 1.002-1.006, $p=0.001$ ) concentrations had worse overall survival.

Patients treated with cisplatin doublets, compared to carboplatin, had better survival (HR 0.613, 95\% CI 0.3890.966, $p=0.035$ ) probably due to selection bias as patients with comorbidities received carboplatin rather than cisplatin. Similarly, patients who received thoracic radiation (HR 0.488 , $95 \%$ CI $0.307-0.776, p=0.002$ ) had better survival. Furthermore, patients who received a PCI had a prolonged survival (HR: 0.446, 95\% CI $0.290-0.686, p<0.0001$ ), which was also significant in multivariate analysis. This result remained significant for the patients without metastases at baseline (HR 0.202 95\% CI: $0.087-0.472, p<0.0001)$ but not for the patients with disseminated disease (HR 0.846, 95\% CI 0.446-1.607, $p=0.51$ ). Patients without metastases who received PCI had a median OS of 23 (95\% CI: 20-26) months vs. 10 (95\% CI: 9-12) months for patients without PCI. PCI was also associated with a longer PFS (HR: 0.156, 95\% CI: 0.055-0.442, $p<0.0001$ ). Eleven patients who were treated with PCI developed cerebral metastases.

\section{Immunohistochemical Markers}

A total of $98 \%$ of the biopsies were positive for CD56, 63\% for chromogranin A, and $80 \%$ for synaptophysin. About $90 \%$ were positive for TTF-1, 100\% for CD44 and Hif-1a, and 84 for GLUT-1 and 72\% for PD-1. The different immunohistochemical markers and $\mathrm{H}$ scores as well as their distribution between metastatic and non-metastatic disease are shown in Tables 2A,B. Only the expression of synaptophysin correlated with a better OS (HR 0.546 95\% CI 0.308-0.966, $p=0.039$ ). Patients without an expression of synaptophysin had a median OS of 12 (95\% CI: 8.115.9) months, whereas patients with a synaptophysin expression had a median OS of 17 months (95\% CI: 13-21) (Figure 2). None of the other markers or the $\mathrm{H}$ scores correlated with a better OS (Table 3). These results were not statistically significant on multivariate analysis (Table 4).

Concerning the PFS, only the expression of TTF-1 (HR 0.286, 95\% CI: $0.117-0.698, p=0.006$, Figure 3) and a GLUT-1 H 
TABLE 2A | Immunhistochemical markers.

\begin{tabular}{|c|c|c|c|c|c|c|c|}
\hline \multirow[b]{2}{*}{ Marker } & \multicolumn{2}{|c|}{ All patients } & \multicolumn{2}{|c|}{ M0 patients } & \multicolumn{2}{|c|}{ M1 patients } & \multirow[t]{2}{*}{$p=0$} \\
\hline & Nr of patients & Nr positiv(\%) & Nr of patients & Nr positiv(\%) & Nr of patients & Nr positiv(\%) & \\
\hline CD56 & 89 & 87 (98\%) & 45 & 45 (100\%) & 44 & 42 (96\%) & 0.99 \\
\hline Chromogranin A & 57 & 36 (63\%) & 27 & 19 (70\%) & 30 & 17 (57\%) & 0.28 \\
\hline Synaptophysin & 79 & 63 (80\%) & 37 & 33 (89\%) & 42 & 30 (71\%) & 0.06 \\
\hline TTF1 & 71 & 64 (90\%) & 41 & $37(90 \%)$ & 30 & 27 (90\%) & 0.97 \\
\hline Hif-1-alpha & 44 & $44(100)$ & 29 & $29(100 \%)$ & 15 & 15 (100\%) & n.a. \\
\hline CD 44 & 44 & $44(100)$ & 29 & $29(100 \%)$ & 15 & 15 (100\%) & n.a. \\
\hline GLUT-1 & 36 & 43 (84\%) & 28 & 25 (89\%) & 15 & $11(73 \%)$ & 0.19 \\
\hline PD-1, & 18 & 13 (72\%) & 12 & 11 (92\%) & 6 & 2 (33\%) & 0.02 \\
\hline PD-L1 tumor & 19 & 2 (10\%) & 13 & $2(15 \%)$ & 6 & $0(0 \%)$ & 0.80 \\
\hline PD-L1 stroma & 19 & $6(32 \%)$ & 13 & 5 (38\%) & 6 & 1 (17\%) & 0.36 \\
\hline PD-L1 macrophages & 19 & 12 (63\%) & 13 & 10 (76\%) & 6 & $2(33 \%)$ & 0.08 \\
\hline MIB-1 & 34 & n.a. & 12 & n.a. & 22 & n.a. & 0.86 \\
\hline
\end{tabular}

n.a., not applicable.

TABLE 2B | H-scores and MIB-1.

\begin{tabular}{lccc}
\hline Biomarker H-Scores & All patients & M0 patients & M1 patients \\
\cline { 2 - 4 } & Median (range) & Median (range) & Median (range) \\
\hline Hif-1a & $190(20-300)$ & $190(20-300)$ & $210(110-270)$ \\
CD 44 & $45(10-260)$ & $40(10-240)$ & $50(20-260)$ \\
GLUT-1 & $50(0-160)$ & $50(0-160)$ & $50(0-140)$ \\
MIB-1 & $70(30-100)$ & $80(30-95)$ & $70(35-100)$ \\
\hline
\end{tabular}

score lower than the median (median $=50, \mathrm{HR}: 0.511,95 \% \mathrm{CI}$ : $0.260-1.003, p=0.05$, Figure 4) correlated with a better PFS.

None of the immunohistochemical markers except for PD 1 (HR 22.000, 95\% CI 1.540-314.292, Table 2A) correlated with the presence of metastases at diagnosis, and the lack of chromogranin A expression correlated with the presence of cerebral metastases (HR 0.250, 95\% CI: 0.079-0.787, $p=0.02$ ).

\section{Radiomic Features}

Our radiomics analysis did not reveal a single texture feature that was highly correlated with overall or progression-free survival. Results are provided in Supplementary Table 3.

Correlation coefficients ranged between -0.48 and 0.39 for overall survival and between -0.46 and 0.38 for progressionfree survival (Spearman correlation coefficients in each case). Figure 5 shows the correlation matrix of the derived radiomics features; the numbers refer to the respective features in Supplementary Table 1.

Highest correlation coefficients for OS were obtained for features original_glcm_Imc1 $(r=-0.48)$, original_glcm_Imc2 $(r=0.40$, high correlation with original_glcm_Imc1 with $r=-0.95$, compare with Figure 5) and original_shape_Maximum2DDiameterColumn $(r=-0.38$, correlation with original_glcm_Imc1 $r=0.41$; these two features were taken for bivariate model building; OS $_{\text {radiomics }}$ ).
The same features were identified for the correlation with progression-free survival, resulting in model PFS $_{\text {radiomics. }}$. Serum NSE and LDH level was added to the feature with strongest correlation (original_glcm_Imc1), resulting in bivariate models OS $_{\text {radiomics-NSE, }}$ OS radiomics-LDH, $_{\text {PFS }}$ radiomics-NSE, and $\mathrm{PFS}_{\text {radiomics }-\mathrm{LDH}}$.

Results of the Cox proportional hazard models are provided in Tables $\mathbf{4}$ and $\mathbf{5}$ for overall survival as an outcome variable. Radiomics features were not able to model survival (both overall and progression free). Hazard ratio was significantly $>1$ for overall survival for NSE and LDH in univariate models; for PFS, $p$-values were 0.10 and 0.11 in univariate models, respectively.

An unpaired Wilcoxon signed rank test was performed to analyze whether the three identified radiomics features were associated with positive or negative synaptophysin / chromogranin A staining, which was negative for both tests $(p>0.05)$.

\section{DISCUSSION}

In the era of personalized medicine, the integration of molecular markers with radiomics features and clinical information to develop novel prognostic biomarkers for treatment response assessment has been the focus of several studies (32-39). Especially in non-small cell lung cancer (NSCLC), the concept of using radiomic features in combination with genomic or proteomic information aiming to enhance our understanding of the tumor phenotype has been reported in several studies (32, 40-42). Radiomics features extracted from CT were related to tumor metabolism, PET tumor stage, and histopathology in NSCLC $(43,44)$, but little is known about their role in SCLC. Similarly, the prognostic and predictive value of different immunohistochemical markers in the treatment response, the presence of metastases, or survival of SCLCs has not been widely investigated. Both IHC and CT have a number of advantages, including being widely available, technically less 


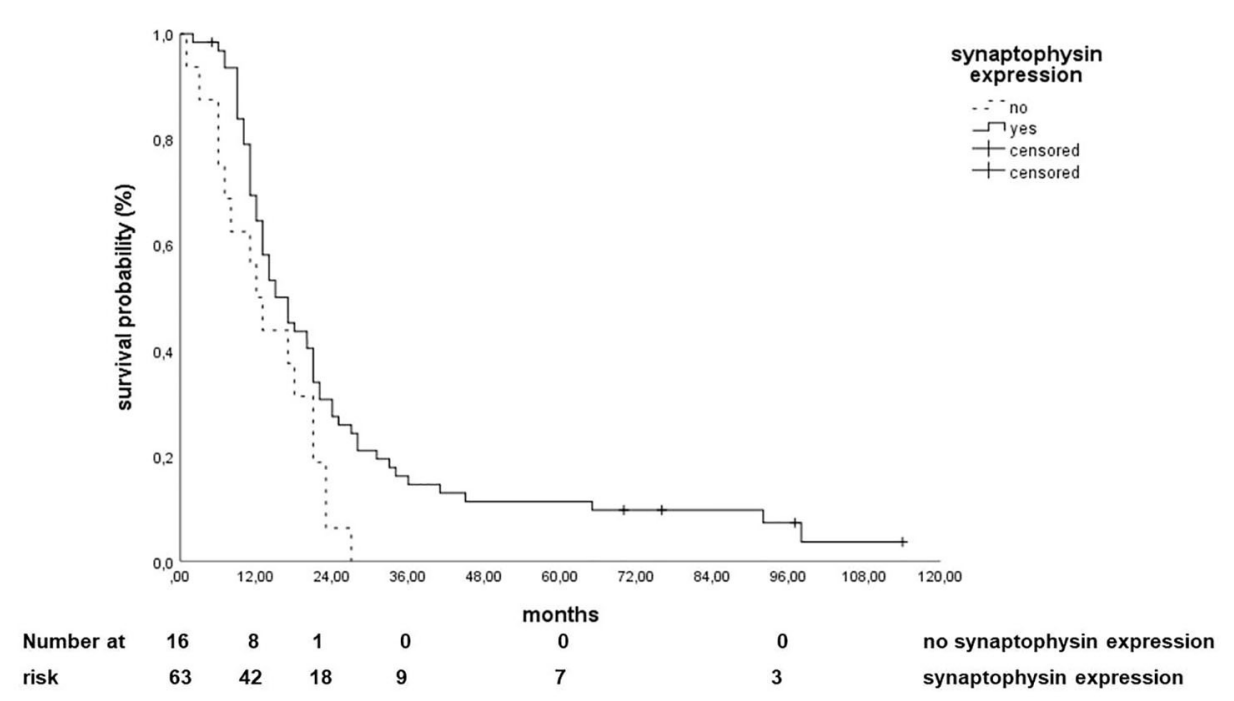

FIGURE 2 | Correlation of synaptophysin expression with overall survival.

TABLE 3 | Univariate analysis of different immunohistochemical markers concerning overall survival.

\begin{tabular}{lcc}
\hline Immunhistochemical marker & Hazard Ratio $(\mathbf{9 5 \%} \mathbf{C l})$ & $\boldsymbol{p}$ \\
\hline CD56 & $0.295(0.071-1.226)$ & 0.09 \\
Chromogranin-A & $0.749(0.430-1.303)$ & 0.31 \\
Synaptophysin & $0.546(0.308-0.966)$ & 0.03 \\
TTF-1 & $0.546(0.247-1.211)$ & 0.14 \\
Hif1a H score & $1.000(0.993-1.006)$ & 0.95 \\
GLUT-1 H score & $1.006(0.998-1.013)$ & 0.13 \\
PD-L1 expression & $0.933(0.829-1.050)$ & 0.25 \\
MIB-1 & $1.001(0.982-1.021)$ & 0.90 \\
\hline
\end{tabular}

TABLE 4 | Multivariate analysis of different parameters concerning overall survival.

\begin{tabular}{lcc}
\hline Variable & Hazard Ratio (95\% Cl) & $\boldsymbol{p}$ \\
\hline $\mathrm{LDH}^{*}$ & $1.001(0.999-1.004)$ & 0.374 \\
$\mathrm{NSE}^{\star}$ & $1.002(0.999-1.005)$ & 0.273 \\
Synaptophysin & $0.914(0.479-1.743)$ & 0.785 \\
$\mathrm{PCl}$ & $0.477(0.265-0.860)$ & 0.014 \\
$\mathrm{M} 1$ & $0.540(0.258-1.129)$ & 0.102 \\
Cisplatin based doublets & $0.555(0.307-1.002)$ & 0.051 \\
Thoracic radiotherapy & $0.532(0.276-1.025)$ & 0.059
\end{tabular}

${ }^{*}$ Continuous variable.

$P C l$, prophylactic cranial irradiation; NSE, neuron specific enolase; $L D H$, lactate dehydrogenase.

challenging, and cost-efficient. Thus, the information derived from both diagnostic tools could enhance our understanding of this aggressive and insufficiently investigated tumor entity.

In our analysis, the lack of synaptophysin expression correlated with a worse OS, contrary to what is reported for
TABLE 5 | Cox-proportional hazard models for radiomics features and radiomics features and serum markers, overall survival as outcome variable.

\begin{tabular}{|c|c|c|}
\hline Feature/Model & Hazard Ratio (95\% Cl) OS & $p$ \\
\hline original_glcm_Imc1 & $142,301(2.141 \mathrm{e}-07-9.457 \mathrm{e}+16)$ & 0.393 \\
\hline original_glcm_Imc2 & $0.1629(0.005-5.219)$ & 0.305 \\
\hline $\begin{array}{l}\text { original_shape_Maximum2D } \\
\text { DiameterColumn }\end{array}$ & $1.01(0.997-1.023)$ & 0.141 \\
\hline NSE & $1.004(1.0-1.007)$ & 0.038 \\
\hline $\mathrm{LDH}$ & $1.003(1.0-1.006)$ & 0.029 \\
\hline \multicolumn{3}{|l|}{ OS radiomics } \\
\hline $\begin{array}{l}\text { original_glcm_Imc1 } \\
\text { and }\end{array}$ & $7518.6(2.666 e-09-2.120 e+16)$ & 0.542 \\
\hline $\begin{array}{l}\text { original_shape_Maximum2D } \\
\text { DiameterColumn }\end{array}$ & 1.009 (9.954e-01-1.023) & 0.191 \\
\hline OS radiomics + NSE & & \\
\hline $\begin{array}{l}\text { original_glcm_Imc1 } \\
\text { and }\end{array}$ & $1.320 e+05(3.801 e-08-4.585 e+17)$ & 0.424 \\
\hline NSE & $1.004(1.0-1.008)$ & 0.035 \\
\hline OS radiomics + LDH & & \\
\hline $\begin{array}{l}\text { original_glcm_Imc1 } \\
\text { and }\end{array}$ & $1.449 \mathrm{e}+06(6.95 \mathrm{e}-08-3.020 \mathrm{e}+19)$ & 0.365 \\
\hline $\mathrm{LDH}$ & $1.003(1.0-1.006)$ & 0.026 \\
\hline
\end{tabular}

NSE: neuron specific enolase, LDH: lactate dehydrogenase, OS: overall survival. Bold values indicate statistically significant.

NSCLCs $(45,46)$, whereas lack of chromogranin A correlated with the presence of cerebral metastases at diagnosis. Both neuroendocrine markers play a significant role in the diagnosis, but little is known about the prognosis of SCLC who lack expression of neuroendocrine markers. Sloman et al. (12) investigated the role of several immunohistochemical markers, including synaptophysin and CD 44 in a small sample of 28 

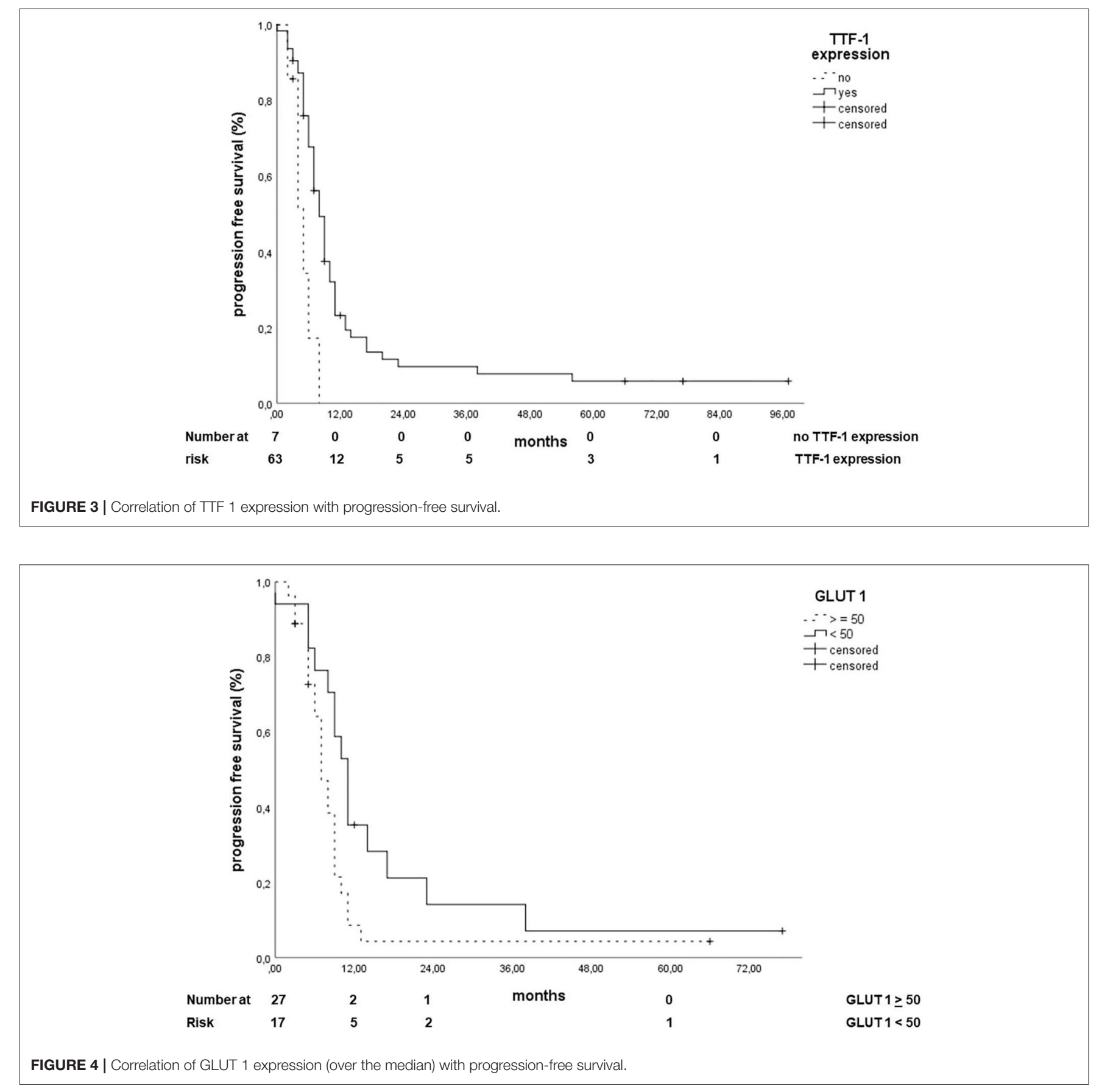

patients, and could not find a significant correlation concerning survival, whereas in an analysis by Drivsholm et al. (47), the expression of chromogranin A in plasma correlated with a worse OS and the stage of disease. Recent studies have shown that SCLC has four different molecular subtypes with a different neuroendocrine character each identified by their key transcriptional regulator $(3,48)$. A subset of small cell lung carcinomas shows loss of the typical neuroendocrine markers. Some of these phenotypes were found to be associated with poor prognosis and chemoresistance although, for other phenotypes, the clinical outcomes have not yet been defined $(48,49)$. A better understanding of the critical signaling pathways operant in particular SCLC subtypes may define vulnerabilities and therapeutic targets $(48,50)$.

The expression of TTF-1 correlated in our analysis with a better progression-free survival. Similar were the results in an analysis by Misch et al. (51) reporting that there was a correlation with the disease control rate (DCR) in patients with metastatic disease (stage IV). There was a significantly $(p=0.006)$ improved response to the treatment in the group of patients with TTF-1expression (DCR 86 vs. 56\%). They concluded that TTF-1 may serve as a predictor of response to first line chemotherapy. 


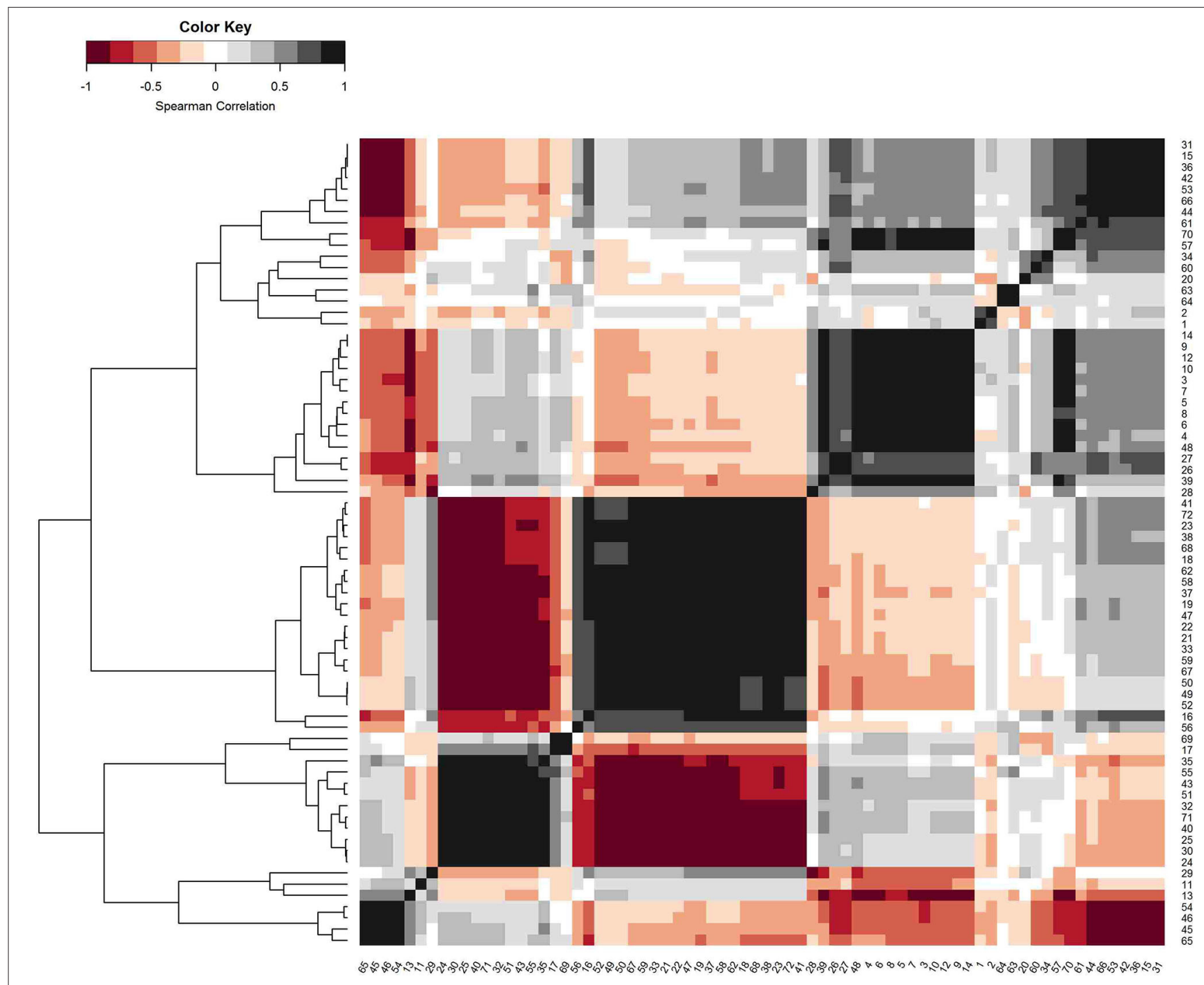

FIGURE 5 | Correlation matrix (Spearman correlation) of the derived radiomics features. Features were ordered with a hierarchical agglomerative clustering algorithm to visualize clusters of collinearity to allow for variable selection.

The proliferation index measured by Ki 67/MIB 1 plays a major role in several malignancies (52-54), but there was no correlation found in our analysis with the OS or with the PFS. Although the Ki-67 protein is well-characterized on the molecular level and extensively used as a proliferation marker, the functional significance still remains unclear. There are indications, however, that a $\mathrm{Ki}-67$ protein expression is an absolute requirement for progression through the cell-division cycle (55). Some studies (56-58) did not find a correlation with response after chemoradiation, similar to our results, but other studies show a correlation of the proliferation rate with the prognosis $(59,60)$.

The uptake of GLUT-1 has been found to be increased in several cancer types $(13,61)$ and is associated with a poorer survival outcome in NSCLC (62). Abnormal expression of GLUT-1 was significantly associated with poor differentiated tumors, positive lymph node metastasis, and larger tumor size, which suggests that overexpression of GLUT1 is linked with enhanced invasive potential, proliferative activity, and decreased patient survival (61). In a study by Ozbudak et al. (14) GLUT-1 was expressed in approximately half of the pulmonary neuroendocrine carcinomas and showed a strong correlation with neuroendocrine differentiation/grade. In our analysis a GLUT-1 H score lower than the median of 50 correlated with a better PFS, but no other association could be found for OS.

The activation of HIF-1a is generally more pronounced in aggressive tumors and can be an independent predictor of poor prognosis in certain types of cancer $(17,63)$. In our analysis, all tumor samples tested were HIF-1a positive. Similarly, 98\% of the biopsies were positive for CD56. This high expression of CD56 in patients with SCLC seemed promising for the use of anti CD56 antibodies for the treatment of CD56 positive tumors, such as Lorvotuzumab mertasine (64). Furthermore, preclinical 
data indicate potent antitumor effects of CD56 chimeric antigen receptor (CAR) T-cells in SCLC (65-67).

The use of immunotherapies in the metastatic setting shows an improvement in OS and PFS in several studies $(21,22)$, but concerning the role of PD-L1 expresion, data are limited as a number of clinical trials with PD-1, PD-L1, or CTLA-4 inhibitors are still ongoing. Initial data from the CheckMate 032 study suggests no correlation between PD-L1 expression and clinical benefit (18). However, in the Keynote-028 and Keynote158 studies of pembrolizumab (anti-PD1), PD-L1 expression, especially combined expression on tumor and immune stromal cells, was associated with improved response to pembrolizumab $(35.7 \%$ vs. $6 \%)(19,20)$. According to Schultheis et al. (68) the PD-1/PD-L1 pathway seems activated in a fraction of SCLCs. In their study, the carcinoma cells were negative in all cases; PD-L1 was expressed in tumor-infiltrating macrophages and was correlated with tumor-infiltrating lymphocytes. In another study, however, Ishii et al. (69) reported that the expression of PD-L1 in tumor cells as defined by $>5 \%$ of positive cells were observed in $71.6 \%$ (73 of 102) SCLC cases. In our analysis, except for a correlation between PD 1 and the presence of metastases, we could not find any correlation between the expression of PD$1 / \mathrm{PD}-\mathrm{L} 1$ and the OS, but results are not easy to interpret due to the very small sample size.

On the other hand, human cancers exhibit strong phenotypic differences that can be visualized non-invasively by medical imaging $(70,71)$. In our explorative radiomics analysis, single radiomics features did correlate moderately with OS and PFS. The small sample size limits the conclusions to be drawn from our findings. However, we decided to only include patients with homogenous CT examinations into the radiomics analysis since image acquisition parameters affect radiomics signatures to a considerable extent (72). In the developed Cox proportional hazard models, radiomics features were not able to significantly model survival while retained conventional serum markers NSE and LDH in the models behaved as expected.

The present study has several limitations, such as its retrospective and single institutional nature, the small sample size as well as a selection bias in the treatments delivered.

In conclusion, a high expression of the neuroendocrine marker synaptophysin correlated significantly with a better survival in patients with SCLC, whereas the expression of TTF-1

\section{REFERENCES}

1. Gazdar AF, Bunn PA, Minna JD. Small-cell lung cancer: what we know, what we need to know and the path forward. Nature Reviews Cancer. (2017) 17:725-37. doi: 10.1038/nrc.2017.87

2. Sabari JK, Lok BH, Laird JH, Poirier JT, Rudin CM. Unravelling the biology of SCLC: implications for therapy. Nat Rev Clin Oncol. (2017) 14:54961. doi: 10.1038/nrclinonc.2017.71

3. Poirier JT, George J, Owonikoko TK, Berns A, Brambilla E, Byers LA, et al. New Approaches to SCLC Therapy: From the Laboratory to the Clinic. $J$ Thorac Oncol. (2020) 15:520-40. doi: 10.1016/j.jtho.2020.01.016

4. Travis WD. Update on small cell carcinoma and its differentiation from squamous cell carcinoma and other non-small cell carcinomas. Mod Pathol. (2012) 25 (Suppl. 1):S18-30. doi: 10.1038/modpathol.2011.150 and a lower GLUT-1 H-score correlated with a better PFS. These results should be further evaluated as synaptophysin-negative tumors might have a poor prognosis and TTF1 negative tumors might be more resistant to current therapies. This might be due to different SCLC-phenotypes, who might profit from treatment intensification. These different markers were not associated with any radiomics features. Furthermore, we did not find a high correlation between single radiomic features with overall survival or progression-free survival as radiomics features were not able to model survival in the analyzed patient subset.

\section{DATA AVAILABILITY STATEMENT}

The datasets generated for this study are available on request to the corresponding author.

\section{ETHICS STATEMENT}

The studies involving human participants were reviewed and approved by Ethic Committee University of Freiburg, Germany. The patients/participants provided their written informed consent to participate in this study.

\section{AUTHOR CONTRIBUTIONS}

EG, MB, BO, FM, SB, UN, and GK: conception and design and collection and assembly of data. EG, MB, BO, SB, UN, and GK: data analysis and interpretation. All authors: manuscript writing and final approval of manuscript.

\section{FUNDING}

This research did not receive any specific grant from funding agencies in the public, commercial, or not-for-profit sectors. MC was funded by Era PERMED call 2018 and BMBF.

\section{SUPPLEMENTARY MATERIAL}

The Supplementary Material for this article can be found online at: https://www.frontiersin.org/articles/10.3389/fonc. 2020.01161/full\#supplementary-material

5. Travis WD. Pathology and diagnosis of neuroendocrine tumors: lung neuroendocrine. Thoracic Surg Clin. (2014) 24:25766. doi: 10.1016/j.thorsurg.2014.04.001

6. Lyda MH, Weiss LM. Immunoreactivity for epithelial and neuroendocrine antibodies are useful in the differential diagnosis of lung carcinomas. Hum Pathol. (2000) 31:980-7. doi: 10.1053/hupa.2000.9076

7. Yesner R. Heterogeneity of so-called neuroendocrine lung tumors. Exp Mol Pathol. (2001) 70:179-82. doi: 10.1006/exmp.2001.2373

8. Zheng G, Ettinger DS, Maleki Z. Utility of the quantitative Ki-67 proliferation index and CD56 together in the cytologic diagnosis of small cell lung carcinoma and other lung neuroendocrine tumors. Acta Cytol. (2013) 57:28190. doi: 10.1159/000346394

9. Holzinger A, Dingle S, Bejarano PA, Miller MA, Weaver TE, Dilauro R, et al. Monoclonal antibody to thyroid transcription factor-1: production, 
characterization, and usefulness in tumor diagnosis. Hybridoma. (1996) 15:49-53. doi: 10.1089/hyb.1996.15.49

10. Lau SK, Luthringer DJ, Eisen RN. Thyroid transcription factor1: a review. Appl Immunohistochem Mol Morphol. (2002) 10:97-102. doi: 10.1097/00129039-200206000-00001

11. Coppola D, Clarke M, Landreneau R, Weyant RJ, Cooper D, Yousem SA. Bcl2, p53, CD44, and CD44v6 isoform expression in neuroendocrine tumors of the lung. Mod Pathol. (1996) 9:484-90.

12. Sloman A, D'amico F, Yousem SA. Immunohistochemical markers of prolonged survival in small cell carcinoma of the lung. An immunohistochemical study. Arch Pathol Lab Med. (1996) 120:465-72.

13. Younes M, Lechago LV, Somoano JR, Mosharaf M, Lechago J. Wide expression of the human erythrocyte glucose transporter Glut1 in human cancers. Cancer Research. (1996) 56:1164-7.

14. Ozbudak IH, Shilo K, Tavora F, Rassaei N, Chu W-S, Fukuoka $\mathrm{J}$, et al. Glucose transporter-1 in pulmonary neuroendocrine carcinomas: expression and survival analysis. Mod Pathol. (2009) 22:633-8. doi: 10.1038/modpathol.2009.6

15. Kaira K, Murakami H, Endo M, Ohde Y, Naito T, Kondo H, et al. Biological correlation of $18 \mathrm{~F}-\mathrm{FDG}$ uptake on pet in pulmonary neuroendocrine tumors. Anticancer Res. (2013) 33:4219-28.

16. Pelosi G, Rodriguez J, Viale G, Rosai J. Typical and atypical pulmonary carcinoid tumor overdiagnosed as small-cell carcinoma on biopsy specimens: a major pitfall in the management of lung cancer patients. Am J Surg Pathol. (2005) 29:179-87. doi: 10.1097/01.pas.0000149690.75462.29

17. Wan J, Chai H, Yu Z, Ge W, Kang N, Xia W, et al. HIF-1alpha effects on angiogenic potential in human small cell lung carcinoma. J Exp Clin Cancer Res. (2011) 30:77. doi: 10.1186/1756-9966-30-77

18. Antonia SJ, López-Martin JA, Bendell J, Ott PA, Taylor M, Eder JP, et al. Nivolumab alone and nivolumab plus ipilimumab in recurrent small-cell lung cancer (CheckMate 032): a multicentre, open-label, phase 1/2 trial. Lancet Oncol. (2016) 17:883-95. doi: 10.1016/S1470-2045(16)30098-5

19. Ott PA, Elez E, Hiret S, Kim DW, Morosky A, Saraf S, et al. Pembrolizumab in patients with extensive-stage small-cell lung cancer: results from the phase Ib KEYNOTE-028 study. J Clin Oncol. (2017) 35:38239. doi: 10.1200/JCO.2017.72.5069

20. Chung HC, Piha-Paul SA, Lopez-Martin J, et al. Pembrolizumab After Two or More Lines of Previous Therapy in Patients With Recurrent or Metastatic SCLC: Results From the KEYNOTE-028 and KEYNOTE-158 Studies. J Thorac Oncol. (2020) 15:618-27. doi: 10.1016/j.jtho.2019.12.109

21. Horn L, Mansfield AS, Szczesna A, Havel L, Krzakowski M, Hochmair MJ, et al. First-line atezolizumab plus chemotherapy in extensive-stage small-cell lung cancer. N Engl J Med. (2018) 379:2220-9. doi: 10.1056/NEJMoa1809064

22. Paz-Ares L, Dvorkin M, Chen Y, Reinmuth N, Hotta K, Trukhin D, et al. Durvalumab plus platinum-etoposide vs. platinum-etoposide in firstline treatment of extensive-stage small-cell lung cancer (CASPIAN): a randomised, controlled, open-label, phase 3 trial. Lancet. (2019) 394:192939. doi: 10.1016/S0140-6736(19)32222-6

23. Lambin P, Rios-Velazquez E, Leijenaar R, Carvalho S, Van Stiphout RG, Granton P, et al. Radiomics: extracting more information from medical images using advanced feature analysis. Eur J Cancer. (2012) 48:4416. doi: 10.1016/j.ejca.2011.11.036

24. Coroller TP, Agrawal V, Huynh E, Narayan V, Lee SW, Mak RH, et al. Radiomic-based pathological response prediction from primary tumors and lymph nodes in NSCLC. J Thorac Oncol. (2017) 12:46776. doi: $10.1016 /$ j.jtho.2016.11.2226

25. Eisenhauer EA, Therasse P, Bogaerts J. New response evaluation criteria in solid tumours: revised RECIST guideline (version 1.1). Eur J Cancer. (2009) 45:228-47. doi: 10.1016/j.ejca.2008.10.026

26. Hirsch FR, Varella-Garcia M, Bunn PAJr, Di Maria MV, Veve R, Bremmes RM, et al. Epidermal growth factor receptor in non-small-cell lung carcinomas: correlation between gene copy number and protein expression and impact on prognosis. J Clin Oncol. (2003) 21:3798-807. doi: 10.1200/JCO.2003. 11.069

27. John T, Liu G, Tsao MS. Overview of molecular testing in non-small-cell lung cancer: mutational analysis, gene copy number, protein expression and other biomarkers of EGFR for the prediction of response to tyrosine kinase inhibitors. Oncogene. (2009) 28 (Suppl. 1):S14-23. doi: 10.1038/onc.2009.197
28. Fedorov A, Beichel R, Kalpathy-Cramer J, Finet J, Fillion-Robin JC, Pujol S, et al. 3D Slicer as an image computing platform for the Quantitative Imaging Network. Magn Reson Imaging. (2012) 30:132341. doi: 10.1016/j.mri.2012.05.001

29. Velazquez ER, Parmar C, Jermoumi M, Mak RH, Van Baardwijk A, Fennessy FM, et al. Volumetric CT-based segmentation of NSCLC using 3D-Slicer. Sci Rep. (2013) 3:3529. doi: 10.1038/srep03529

30. Van Griethuysen JJM, Fedorov A, Parmar C, Hosny A, Aucoin N, Narayan V, et al. Computational radiomics system to decode the radiographic phenotype. Cancer Res. (2017) 77:e104-7. doi: 10.1158/0008-5472.CAN-17-0339

31. Schneider A, Hommel G, Blettner M. Linear regression analysis: part 14 of a series on evaluation of scientific publications. Dtsch Arztebl Int. (2010) 107:776-82. doi: 10.3238/arztebl.2010.0776

32. Aerts HJ, Grossmann P, Tan Y, Oxnard GR, Rizvi N, Schwartz LH, et al. Defining a radiomic response phenotype: a pilot study using targeted therapy in NSCLC. Sci Rep. (2016) 6:33860. doi: 10.1038/srep33860

33. Dinapoli N, Casà C, Barbaro B, Chiloiro GV, Damiani A, Di Matteo M, et al. Radiomics for rectal cancer. Transl Cancer Res. (2016) 5:42431. doi: $10.21037 /$ tcr.2016.06.08

34. Gillies RJ, Kinahan PE, Hricak H. Radiomics: images are more than pictures, they are data. Radiology. (2016) 278:563-77. doi: 10.1148/radiol.2015151169

35. O'connor JP, Aboagye EO, Adams JE, Aerts HJ, Barrington SF, Beer AJ, et al. Imaging biomarker roadmap for cancer studies. Nat Rev Clin Oncol. (2017) 14:169-86. doi: 10.1038/nrclinonc.2016.162

36. Bae S, Choi YS, Ahn SS, Chang JH, Kang SG, Kim EH, et al. Radiomic MRI phenotyping of glioblastoma: improving survival prediction. Radiology. (2018) 289:797-806. doi: 10.1148/radiol.2018180200

37. Bonekamp D, Kohl S, Wiesenfarth M, Schelb P, Radtke JP, Gotz M, et al. Radiomic machine learning for characterization of prostate lesions with MRI: comparison to ADC values. Radiology. (2018) 289:12837. doi: 10.1148/radiol.2018173064

38. Story MD, Durante M. Radiogenomics. Med Phys. (2018) 45:e111122. doi: $10.1002 / \mathrm{mp} .13064$

39. Forghani R, Savadjiev P, Chatterjee A, Muthukrishnan N, Reinhold C, Forghani B. Radiomics and artificial intelligence for biomarker and prediction model development in oncology. Comp Struc Biotechnol J. (2019) 17:9951008. doi: 10.1016/j.csbj.2019.07.001

40. Grossmann P, Stringfield O, El-Hachem N, Bui MM, Rios Velazquez E, Parmar $\mathrm{C}$, et al. Defining the biological basis of radiomic phenotypes in lung cancer. Elife. (2017) 6:e19993. doi: 10.7554/eLife.23421.027

41. Bak SH, Park H, Lee HY, Kim Y, Kim HL, Jung SH, et al. Imaging genotyping of functional signaling pathways in lung squamous cell carcinoma using a radiomics approach. Sci Rep. (2018) 8:3284. doi: 10.1038/s41598-018-21706-1

42. Kalra M, Wang G, Orton CG. Radiomics in lung cancer: its time is here. Med Phys. (2018) 45:997-1000. doi: 10.1002/mp.12685

43. Avanzo M, Stancanello J, El Naqa I. Beyond imaging: the promise of radiomics. Phys Med. (2017) 38:122-39. doi: 10.1016/j.ejmp.2017.05.071

44. Zhou M, Leung A, Echegaray S, Gentles A, Shrager JB, Jensen KC, et al. Nonsmall cell lung cancer radiogenomics map identifies relationships between molecular and imaging phenotypes with prognostic implications. Radiology. (2018) 286:307-15. doi: 10.1148/radiol.2017161845

45. Pelosi G, Pasini F, Sonzogni A, Maffini F, Maisonneuve P, Iannucci A, et al. Prognostic implications of neuroendocrine differentiation and hormone production in patients with Stage I nonsmall cell lung carcinoma. Cancer. (2003) 97:2487-97. doi: 10.1002/cncr.11376

46. Feng J, Sheng H, Zhu C, Qian X, Wan D, Su D, et al. Correlation of neuroendocrine features with prognosis of non-small cell lung cancer. Oncotarget. (2016) 7:71727-36. doi: 10.18632/oncotarget.12327

47. Drivsholm L, Paloheimo LI, Østerlind K. Chromogranin A, a significant prognostic factor in small cell lung cancer. Br J Cancer. (1999) 81:66771. doi: $10.1038 /$ sj.bjc. 6690745

48. Rudin CM, Poirier JT, Byers LA, Dive C, Dowlati A, George J, et al. Molecular subtypes of small cell lung cancer: a synthesis of human and mouse model data. Nat Rev Cancer. (2019) 19:289-97. doi: 10.1038/s41568-019-0133-9

49. Gazdar AF, Carney DN, Nau MM, Minna JD. Characterization of variant subclasses of cell lines derived from small cell lung cancer having distinctive biochemical, morphological, and growth properties. Cancer Res. (1985) 45:2924-30. 
50. Sonkin D, Thomas A, Teicher BA. Are neuroendocrine negative small cell lung cancer and large cell neuroendocrine carcinoma with WT RB1 two faces of the same entity? Lung Cancer Manag. (2019) 8:Lmt13. doi: 10.2217/lmt-2019-0005

51. Misch D, Blum T, Boch C, Weiss T, Crolow C, Griff S, et al. Value of thyroid transcription factor (TTF)-1 for diagnosis and prognosis of patients with locally advanced or metastatic small cell lung cancer. Diagn Pathol. (2015) 10:21. doi: 10.1186/s13000-015-0250-z

52. Martin B, Paesmans M, Mascaux C, Berghmans T, Lothaire P, Meert AP, et al. Ki-67 expression and patients survival in lung cancer: systematic review of the literature with meta-analysis. Br J Cancer. (2004) 91:201825. doi: 10.1038/sj.bjc. 6602233

53. Chen X, He C, Han D, Zhou M, Wang Q, Tian J, et al. The predictive value of Ki-67 before neoadjuvant chemotherapy for breast cancer: a systematic review and meta-analysis. Future Oncol. (2017) 13:84357. doi: 10.2217/fon-2016-0420

54. Yoshikawa K, Shimada M, Higashijima J, Nakao T, Nishi M, Takasu C, et al. Ki-67 and survivin as predictive factors for rectal cancer treated with preoperative chemoradiotherapy. Anticancer Res. (2018) 38:17359. doi: 10.21873/anticanres.12409

55. Scholzen T, Gerdes J. The Ki-67 protein: from the known and the unknown. J Cell Physiol. (2000) 182:311-22. doi: 10.1002/SICI10974652200003182:3<311::AID-JCP1>3.0.CO;2-9

56. Paik KH, Park YH, Ryoo B-Y, Yang SH, Lee JC, Kim CH, et al. Prognostic value of immunohistochemical staining of p53, bcl-2, and ki-67 in small cell lung cancer. J Kor Med Sci. (2006) 21:35-9. doi: 10.3346/jkms.2006.21.1.35

57. Erler BS, Presby MM, Finch M, Hodges A, Horowitz K, Topilow AA, et al. CD117, Ki-67, and p53 predict survival in neuroendocrine carcinomas, but not within the subgroup of small cell lung carcinoma. Tumour Biol. (2011) 32:107-11. doi: 10.1007/s13277-010-0104-y

58. Ishibashi $\mathrm{N}$, Maebayashi $\mathrm{T}$, Aizawa $\mathrm{T}$, Sakaguchi $\mathrm{M}$, Nishimaki $H$, Masuda S. Correlation between the Ki-67 proliferation index and response to radiation therapy in small cell lung cancer. Radiat Oncol. (2017) 12:16. doi: 10.1186/s13014-016-0744-1

59. Bohm J, Koch S, Gais P, Jutting U, Prauer HW, Hofler H. Prognostic value of MIB-1 in neuroendocrine tumours of the lung. J Pathol. (1996) 178:4029. doi: 10.1002/SICI1096-9896199604178:4<402::AID-PATH498>3.0.CO;2-5

60. Kayser G, Gunzenhauser I, Becker HD, Herth F, Kayser K. [Proliferation rate of small cell lung carcinoma. A cytomorphometric parameter with prognostic significance]. Pathologe. (2001) 22:326-32. doi: 10.1007/s002920100480

61. Yu M, Yongzhi H, Chen S, Luo X, Lin Y, Zhou Y, et al. The prognostic value of GLUT1 in cancers: a systematic review and meta-analysis. Oncotarget. (2017) 8:43356-67. doi: 10.18632/oncotarget.17445

62. Zhang B, Xie Z, Li B. The clinicopathologic impacts and prognostic significance of GLUT1 expression in patients with lung cancer: a metaanalysis. Gene. (2019) 689:76-83. doi: 10.1016/j.gene.2018.12.006

63. Zagzag D, Zhong H, Scalzitti JM, Laughner E, Simons JW, Semenza GL. Expression of hypoxia-inducible factor lalpha in brain tumors: association with angiogenesis, invasion, and progression. Cancer. (2000) 88:260618. doi: 10.1002/1097-01422000060188:11<2606::AID-CNCR25>3.0.CO;2-W

64. Shah MH, Lorigan P, O'brien ME, Fossella FV, Moore KN, Bhatia S, et al. Phase I study of IMGN901, a CD56-targeting antibody-drug conjugate, in patients with CD56-positive solid tumors. Invest New Drugs. (2016) 34:2909. doi: 10.1007/s10637-016-0336-9

65. Levine BL. Performance-enhancing drugs: design and production of redirected chimeric antigen receptor (CAR) T cells. Cancer Gene Ther. (2015) 22:79-84. doi: 10.1038/cgt.2015.5

66. Rosenberg SA, Restifo NP. Adoptive cell transfer as personalized immunotherapy for human cancer. Science. (2015) 348:628. doi: $10.1126 /$ science.aaa4967

67. Bunn PAJr, Minna JD, Augustyn A, Gazdar AF, Ouadah Y, Krasnow MA, et al. Small cell lung cancer: can recent advances in biology and molecular biology be translated into improved outcomes? J Thorac Oncol. (2016) 11:45374. doi: 10.1016/j.jtho.2016.01.012

68. Schultheis AM, Scheel AH, Ozretic L, George J, Thomas RK, Hagemann T, et al. PD-L1 expression in small cell neuroendocrine carcinomas. Eur J Cancer. (2015) 51:421-6. doi: 10.1016/j.ejca.2014.12.006

69. Ishii H, Azuma K, Kawahara A, Yamada K, Imamura Y, Tokito T, et al. Significance of programmed cell death-ligand 1 expression and its association with survival in patients with small cell lung cancer. J Thorac Oncol. (2015) 10:426-30. doi: 10.1097/JTO.0000000000000414

70. Aerts HJ, Velazquez ER, Leijenaar RT, Parmar C, Grossmann P, Carvalho S, et al. Decoding tumour phenotype by noninvasive imaging using a quantitative radiomics approach. Nat Commun. (2014) 5:4006. doi: $10.1038 /$ ncomms5006

71. Zhang Y, Oikonomou A, Wong A, Haider MA, Khalvati F. Radiomicsbased prognosis analysis for non-small cell lung cancer. Sci Rep. (2017) 7:46349. doi: 10.1038/srep46349

72. Berenguer R, Pastor-Juan MDR, Canales-Vazquez J, Castro-Garcia M, Villas MV, Mansilla Legorburo F, et al. Radiomics of CT features may be nonreproducible and redundant: influence of CT acquisition parameters. Radiology. (2018) 288:407-15. doi: 10.1148/radiol.2018 172361

Conflict of Interest: The authors declare that the research was conducted in the absence of any commercial or financial relationships that could be construed as a potential conflict of interest.

Copyright (c) 2020 Gkika, Benndorf, Oerther, Mohammad, Beitinger, Adebahr, Carles, Schimek-Jasch, Zamboglou, Frye, Bamberg, Waller, Werner, Grosu, Nestle and Kayser. This is an open-access article distributed under the terms of the Creative Commons Attribution License (CC BY). The use, distribution or reproduction in other forums is permitted, provided the original author(s) and the copyright owner(s) are credited and that the original publication in this journal is cited, in accordance with accepted academic practice. No use, distribution or reproduction is permitted which does not comply with these terms. 